\title{
Correction to: A survey of challenges for runtime verification from advanced application domains (beyond software)
}

\section{César Sánchez ${ }^{1}$. Gerardo Schneider ${ }^{2}$. Wolfgang Ahrendt ${ }^{3}$. Ezio Bartocci ${ }^{4}$.} Domenico Bianculli ${ }^{5}$. Christian Colombo ${ }^{6}$. Yliès Falcone ${ }^{7}$. Adrian Francalanza ${ }^{6}$. Srdan Krstić ${ }^{8}$. João M. Lourenço ${ }^{9}$. Dejan Nickovic ${ }^{10}$. Gordon J. Pace ${ }^{6}$. Jose Rufino ${ }^{11}$. Julien Signoles ${ }^{12}$. Dmitriy Traytel $^{8} \cdot$ Alexander Weiss $^{13}$

Published online: 15 November 2019

๑) Springer Science+Business Media, LLC, part of Springer Nature 2019

\section{Correction to: Formal Methods in System Design https://doi.org/10.1007/s10703-019-00337-w}

The correct name of the seventh author is Yliès Falcone.

His correct affiliation is Univ. Grenoble Alpes, Inria, CNRS, Grenoble INP, LIG, 38000 - Grenoble, France

The original article has been updated.

Publisher's Note Springer Nature remains neutral with regard to jurisdictional claims in published maps and institutional affiliations.

The original article can be found online at https://doi.org/10.1007/s10703-019-00337-w.

\section{César Sánchez}

cesar.sanchez@imdea.org

1 IMDEA Software Institute, Madrid, Spain

2 University of Gothenburg, Göteborg, Sweden

3 Chalmers University of Technology, Göteborg, Sweden

4 TU Wien, Vienna, Austria

5 University of Luxembourg, Luxembourg City, Luxembourg

6 University of Malta, Msida, Malta

7 Univ. Grenoble Alpes, Inria, CNRS, Grenoble INP, LIG, 38000 Grenoble, France

8 ETH Zürich, Zurich, Switzerland

9 Universidade Nova de Lisboa, Lisbon, Portugal

10 Austrian Institute of Technology, Seibersdorf, Austria

11 Universidade de Lisboa, Lisbon, Portugal

12 CEA LIST, Software Reliability and Security Lab, Palaiseau, France

13 Accemic Technologies GmbH, Kiefersfelden, Germany 